\title{
BARTOLOMÉ DE AMPHOUX: PROYECTO DE CUARTEL Y PUENTE PARA ARCOS DE LA FRONTERA ${ }^{1}$
}

\author{
POR José Manuel BaEna Gallé
}

El siglo XVIII es un período de gran vitalidad y apogeo para toda Andalucía. Prueba de ello es el desarrollo y crecimiento de algunas poblaciones que adquieren una vitalidad inusitada hasta ese momento. Una de ellas fue Arcos de la Frontera, ciudad que pertenecía al Reino de Sevilla, dependiente de los Ponce de León, quienes ostentaban el titulo de Duques de Arcos. Ejemplo de este período floreciente para la población es el Catastro de Ensenada, fechado en Sevilla en diciembre de 1756, donde se indica que el total de vecinos era de 3000 . Pero tal vez más significativo sea la gran actividad constructiva que se observa en esta época, actividad que continúa y aumenta la tradición y esfuerzos de etapas anteriores. En este sentido cabe citar la restauración de la Iglesia de Santa María en 1713 y el proyecto de ampliación de San Pedro en 1714, ambas ideadas por Lorenzo Fernández de Iglesias e informadas favorablemente por Diego Antonio Díaz, o el proyecto de Vicente Catalán Bengoechea para la torre de Santa María, inconcluso pero muy ambicioso, de 1758, o la construcción, por parte de Pedro de Silva, de la torre-fachada de San Pedro ${ }^{2}$.

Una de las razones que podían explicar este crecimiento y desarrollo es la posición privilegiada de la población. Arcos se asienta sobre una peña en el centro de la campiña, dominando la vega del río Guadalete y dando paso a la serranía gaditana y malagueña. Se trata de un nudo vital de comunicaciones, de

1. El presente texto es una revisión y actualización de la comunicación presentada a las Jomadas Nacionales "La ingeniería militar en la cultura artística española". Cádiz, noviembre 1989.

2. FALCON MÁRQUEZ, Teodoro: Iglesias de la Sierra de Cádiz. Cádiz, 1983. Pp. 23-24-35. 
alto valor estratégico para la actividad militar y defensa de la zona, como más tarde veremos, y de lo que es consciente el propio Concejo de Arcos que llega a afirmar que "Dista esta ciudad cinco leguas dela de xerez, siete dela del puerto de santa maria y porella ala de cadiz nuebe y doze por tierra y ser el paso para la de malaga; Antequera y demas ciudades Villas y lugares del Reyno de Granada y Campo de Gibraltar" ${ }^{3}$. Esta circunstancia es la que aconsejará el establecimiento de un cuartel en la población para cuya construccion se elaboran varios informes y un proyecto. Precisamente de este se trata a continuación.

Dicho proyecto se recoge en un plano, firmado en Cádiz el 4 de febrero de 1744 por Bartolomé de Amphoux. Fue dado a conocer por Marzal, pero sin profundizar en su estudio ni en el de la documentación que lo acompaña ${ }^{4}$. El plano se conserva en el Archivo General de Simancas ${ }^{5}$ y consta de tres partes claramente diferenciadas: un plano de la ciudad, un proyecto de cuartel y un proyecto de puente. De dichas partes se hará un estudio a continuación, pero primeramente hay que señalar y resaltar algunos aspectos destacados de los dibujos. La importancia de esta documentación radica, en primer lugar, en que ofrece el plano mas antiguo conocido de la ciudad y, en segundo término, en que aporta el proyecto de un puente, vieja aspiración de la ciudad, que no se verá cumplido hasta el siglo XIX.

Con respecto al plano de la ciudad se plantean varios inconvenientes, ya que sólo muestra una parte de un barrio de la ciudad -el llamado Barrio de San Diego- y no de todo el conjunto urbano. Además, se trata de un dibujo esquemático, pues solo da una serie de referencias de algunos edificios y de su situación

\footnotetext{
3. Archivo General de Simancas (A.G.S.), Leg. 3513. Acuerdo capitular de 5 de agosto de 1743.

4. MARZAL MARTÍNEZ, Amparo: "Notas sobre arquitectura militar dieciochesca en Andalucía. Cuarteles". En Actas del I Congreso de Historia de Andalucía. Volumen IV. Andalucía Moderna. Siglo XVIII. Tomo II. Córdoba, 1978. p. 19.

5. ALVAREZ TERAN, María Concepción: Mapas, planos y dibujos (años 1503-1805). Catalogo XXIX. Volumen I. Archivo General de Simancas. Valladolid, 1980. P. 40.

"Plano que demuestra una porcion del Barrio de Sn. Diego de la ciudad de Arcos en cuyo sitio se puede plantificar un Quartel para un regimiento de Cavalleria que en su defecto podra servir para un Batallon de Infanteria ..."

Escala de $75 \mathrm{mms}$. las 100 varas.

"Planos y perfiles de un Quartel para un regimiento de Cavalleria con su Pavellon para los correspondientes oficiales que se propone construir en la Ciudad de Arcos ..."

"Fachada del Puente proyectado", todos por D.B. Amphoux.

Cádiz, 4 de febrero de 1744.

Escalas para los Planos, Perfiles y Fachada del Puente de 155 mms. las 20 toesas y de 150 mms. las 45 varas.

Tinta y colores a la aguada. Con explicación.

365 x 1900 mms. Recuadro de 345 x 1885 mms.

A.G.S. M.P. y D. XXII-72. G.M., Leg. 3513.
} 
en la zona en el siglo XVIII. Esto es debido a que el plano es solamente un dibujo informativo, realizado por un ingeniero militar para estudiar y demostrar cuál debía ser la ubicación más adecuada de un cuartel en la ciudad. Es importante señalar, que el terreno que aparece en el plano es el que actualmente es conocido como Barrio Bajo, por estar situado entre la peña y el cauce del río, no constando en ninguna de las fuentes consultadas el nombre de San Diego; sin embargo sí se sabe que al menos desde 1770 existe la denominación de Barrio de Abajo. Es una zona que empezó a surgir como parte integrante de Arcos a fines del siglo XVIII y principios del XIX y que mantuvo siempre unos caracteres artesanales y fabriles muy acusados. Hasta entonces no había sido más que una zona extramuros de la ciudad, y su importancia radicará en la incipiente industria aceitera y de curtidos que más tarde, favorecida por la proximidad del río, hará crecer el barrio ${ }^{6}$.

Los aspectos del plano que dan referencias sobre la situación de la zona en aquella época son de dos tipos. Por un lado naturales, ya que se señalan los principales elementos geográficos existentes, como son el río Guadalete, que circunda la peña sobre la que esta asentada la ciudad, y el Cerro del Calvario, especie de promontorio junto al llano de la Caridad. Por otro lado arquitectónicos, donde destacan, primero, los edificios religiosos, como la Caridad y su Cementerio, el Calvario y San Diego, que muestran la importancia que tienen las instituciones religiosas y sus construcciones para la configuración urbanística de una zona, pues sirven de centro de atracción de la vida ciudadana. Por otra parte, está lo que se puede llamar arquitectura industrial, centrada en los molinos y que, como anteriormente se dijo, es la que va dando vida al barrio y la razón de su crecimiento. Por último, se encuentran lo que podría denominarse como elementos de infraestructura ciudadana: la red de abastecimiento de agua y la de comunicaciones.

Los edificios religiosos señalados fueron las primeras construcciones conocidas del barrio. Alrededor de ellas fue surgiendo todo el núcleo poblacional, siendo claro ejemplo de esto el llamado Convento de San Diego, pudiendo ser el de franciscanos descalzos. Se sabe que desde fines del siglo XVI existía allí una ermita, de la Virgen de la Cabeza, y que en torno a 1603 se fundó un convento cuya obra se terminó en 1644. Se eligió para su construccion la zona de extramuros y por las fechas sería, probablemente, el primer edificio de carácter público del barrio. Otro elemento de tipo religioso que figura en el dibujo es el Calvario, situado en el cerro del mismo nombre, que no era más que el lugar de un humilladero con una cruz, la cual sería similar a la que hay en la plaza de la Caridad, donde culminaba un Vía Crucis que salía desde la Iglesia de San Pedro,

6. CUEVAS, José y Jesús de las: Arcos de la Frontera. Jerez, 1968. P. 238. 
situada en la parte alta de la ciudad ${ }^{7}$. La última construcción religiosa que se observa es la Ermita de la Caridad, que fue erigida alrededor del año 1600. Había allí una cofradía o hermandad que se dedicaba a dar sepultura a los ajusticiados en la ciudad o a los extranjeros muertos por la zona. En la época del plano aun era una sencilla ermita, ya que será don Manuel Ayllón de Lara, nombrado prioste en 1740, quien mandará construir el Asilo y su iglesia en 1757. Un elemento diferenciado en el plano es el Camposanto de la Caridad, que tiene gran importancia para la evolución urbanística de la zona, puesto que ha configurado la actual Plaza, en la que en recuerdo de su antigua función, se erige una cruz.

Por lo que respecta a la arquitectura industrial, destacan los molinos, de los que existen varios ejemplos en el plano: el molino de Bohorques, el de don Gregorio del Ara, el de las Monjas, el de San Juan de Dios, el de la Fábrica de San Pedro, y el de la Fábrica de Santa María, éste en la otra orilla del río. Es destacable el hecho de que los principales centros religiosos de la ciudad tuviesen un molino propio, lo que nos vuelve a hablar de la importancia de este tipo de instituciones en la España del Antiguo Régimen. Como ya se ha visto, el Barrio Bajo surge en esta época con gran fuerza, gracias a lo que podría denominarse como industria de transformación agraria; se situó en esta zona por la proximidad al río, cuya fuerza motriz es necesaria para realizar las diferentes labores. Existen en el barrio molinos de aceite, cantarerías, fábricas de harinas, de ladrillos, de orujo, y sobre todo, fábricas de curtidos ${ }^{8}$. Por todo ello puede afirmarse que la economía de tipo industrial de la zona era muy floreciente en esta época y que la misma irá configurando el barrio como zona pujante y de creciente importancia.

Un último aspecto es el del abastecimiento de aguas. El problema del agua es siempre un conflicto para cualquier núcleo de población en expansión. En el plano solo hay referencia a la existencia de una fuente, llamada de "las mugeres" -que puede ser la noria a la que aluden todas las obras consultadas-, situada a la orilla del río, y alejada del núcleo construido del barrio, por lo que se deduce que este carecía de un adecuado abastecimiento. Las autoridades arcenses son conscientes de eso y por ello al solicitar el cuartel, proponen la construcción de una fuente en la plaza del Convento de los Descalzos, noticia que confirma la idea de que este edificio religioso era el núcleo central del barrio. El agua para esta fuente seria traída desde la de la Plata, distante unos 1500 metros, ya que debido

7. Véase: PEREZ REGORDAN, Manuel: Las calles de Arcos. Estudio histórico, anecdótico, estadístico y religioso. Arcos de la Frontera, 1975. p. 38.

8. CUEVAS: Op. cit., p. 238. 
a su altura se podía hacer la conducción a través de cañerías ${ }^{9}$. Esta obra se pensaba incluir en el presupuesto del cuartel ${ }^{10}$. El otro aspecto de la infraestructura, las comunicaciones, se evidencia con el propio proyecto de puente.

Con respecto al cuartel el primer problema a tratar es la necesidad del mismo. Ya se vio que la causa principal que se alegaba para su construcción era la situación estratégica de la ciudad, lo que hacía habitual el paso de contingentes de tropas, camino de alguna otra plaza "'. Con la aparición del estado considerado "moderno" en el siglo XVIII, surgen nuevas necesidades, una de las cuales era la de un cuerpo de defensa estable y permanente, es decir, un ejército profesional que permita una defensa eficaz del territorio. Esto traía consigo problemas, uno de los cuales era el del alojamiento de los soldados. Así aparece el cuartel como un nuevo tipo de construcción. Anteriormente el alojamiento de los soldados se realizaba en mesones o casas deshabitadas y, en su defecto, en las casas de los vecinos, pero siempre las costas eran a cargo de los habitantes de la ciudad. Esto suscitaba numerosos problemas, ya que la situación era incómoda tanto para el soldado como para los habitantes del lugar ${ }^{12}$. Prueba de ello son las numerosas quejas de los vecinos sobre el comportamiento de las tropas, que a veces llegaba a ser realmente bárbaro ${ }^{13}$. Así, va surgiendo la necesidad de un cuartel donde se alojen los soldados sin que se moleste a la ciudad y donde las tropas pudiesen estar mas cómodas y, a la vez, agrupadas.

En España, es en el siglo XVIII cuando se plantea toda esta problemática a causa de la política de reformas de la nueva dinastía borbónica. El alojamiento de los soldados debía ser algo programado y racional como correspondía a una nación moderna ${ }^{14}$. Hay obras teóricas que establecen una tipología para la construcción de cuarteles como por ejemplo, el Proyecto General Impreso de 8 de abril de 1718 de Jorge Próspero Verboom en el que se establece un tipo de cuartel, basado en el que el mariscal francés Vaubam había diseñado para la

9. Esta fuente se encontraba próxima a la ermita de San Antonio del Valle; su nombre le venía dado por la arena fina y blanca que se obtenía en la zona y que se exportaba a Sevilla para limpiar y labrar la plata. Cfr.: GAMAZA ROMERO, Pedro: Descripcion de la Muy Noble y Leal Ciudad de Arcos de la Frontera. Jerez, 1640 (Ed.: Arcos, 1902, p. 179).

10. Acuerdo municipal de 5 de agosto de 1743. A.G.S. Leg. 3513.

11. Esto lo afirman las autoridades arcenses “... son muy continuados los tranzitos de Infanteria y cavalleria...". A.G.S. Leg. 3513. Acuerdo municipal de 5 de agosto de 1743.

12. Así el cabildo municipal de Arcos opina que “... por no haber quartel sealoxan en cassas de Paysanos conel quebranto que sedaxa considerar deque resulta bastantes desasones y para evitarlas sea visto presisada la ciudad a ponerlos en Mezones enque atenido excesivos Gastos y los soldados no están con la comodidad que serrequiere ...".

13. Por ejemplo en Arcos, donde el paso del Regimiento de Dragones de Pavía el 29 y 30 de septiembre de 1720, provocó mas de 40 muertos. Cfr.: CUEVAS, Op. Cit., p. 72.

14. MARZAL MARTÍNEZ, Op. Cit., p. 4. 
infantería de su país en el siglo XVII. El cuartel se va diseñando como un lugar no solo para la guerra, sino también para la paz. Por ello se irá independizando de las fortificaciones y se configurará como un edificio urbano individualizado y plenamente integrado en la vida ciudadana. Así, las poblaciones irán solicitando la construccion de cuarteles, por las necesidades antes señaladas, pero también impulsados por la legislación que lo favorece ${ }^{15}$. Marzal indica que solo en Andalucía, entre 1733 y 1769 , se solicitaron veinticinco cuarteles, pero que solo se realizaron proyectos para seis y que al final únicamente se construyeron dos ${ }^{16}$.

Toda esta política constructiva se basa en un hecho muy importante, como es la creación en 1711 del Cuerpo de Ingenieros Militares por Felipe V, que viene a ser el primer cuerpo organizado de técnicos que posee España. Estos se forman en la práctica, pero también en la teoría de los tratados de matemáticas y de fortificación y son los que difunden e introducen en el país las ideas del Siglo de las Luces. Se dedican a las labores propias de la fortificación pero también destacan por su actuación en el campo de las obras civiles (caminos, puentes, canales, etc.) y religiosas y en tareas de ordenación espacial y urbanística. Por todo ello es posible afirmar que los miembros de este cuerpo son los verdaderos protagonistas de la más moderna política constructiva del siglo XVIII ${ }^{17}$.

Precisamente uno de estos ingenieros es el autor del proyecto de cuartel de Arcos. Se trata de Bartolomé Amphoux y Perelló, quien desde 1738 se encontraba en Cádiz, donde alcanzo el empleo de Teniente Coronel ${ }^{18}$. Realizó varios proyectos -un cuartel en Vejer de la Frontera; muelles, murallas, baterías y cuarteles de Cádiz, así como modificaciones a los proyectos de Ignacio Sala para la Aduana, Consulado y Casa de Contratación de Cádiz-, antes de pasar a América, donde continuó su labor.

15. Por ejemplo, el Real Decreto de 16 de febrero de 1748 que aprueba una rebaja en la contribución de la Sal y destino del valimiento de arbitrios a la fabrica de cuarteles. Cfr.: MARZAL MARTÍNEZ: Op. Cit., p. 6.

16. MARZAL MARTÍNEZ: $O p$. Cit., p. 6-7. Existe un estudio más profundo de los cuarteles proyectados para la provincia de Huelva. Véase: HERNÁNDEZ, Juan Carlos: "Gerónimo Amici y los proyectos de cuarteles para el regimiento de caballería de Andalucía, en la provincia de Huelva". Espacio, Tiempo y Forma. Serie VII. Historia del Arte, t. 4. Madrid, 1991. Pp. 239-264.

17. Véase: CAPEL, Horacio y otros: Los ingenieros militares en España. Siglo XVIII. Repertorio biográfico e inventario de su labor científica y espacial. Barcelona, 1983. Pp. 6-7.

18. CAPEL: Op. Cit., pp. 34-35. En esta obra se menciona el proyecto de cuartel de Arcos, pero situándolo en el barrio de San Pedro. Sin duda se trata de un error, pues el espacio urbano, conocido popularmente como Barrio Bajo, se denominaba de San Diego, nombre con el que se le cita en la leyenda del Plano. Por otra parte, hay que señalar que la localización del auténtico barrio de San Pedro en la parte alta de la ciudad, hace imposible establecer una obra de estas características por falta de espacio.

Véase también: CAPEL, Horacio, SÁNCHEZ, Joan Eugeni, MONCADA, Omar: De Palas a Minerva. La formación cientifica y la estructura institucional de los ingenieros militares en el siglo XVIII. Barcelona, 1988. 
Aduciendo las razones antes expuestas, el Ayuntamiento de Arcos solicitó la construccion del cuartel. Para llevarlo a cabo pidió autorización al Marqués de la Ensenada para establecer una serie de arbitrios sobre la carne, el vino, el aceite y el vinagre, con los que hacer frente a las obras ${ }^{19}$. Estos fueron concedidos el 1 de septiembre de 1743 , estimándose las ganancias en 13.717 reales y 12 maravedís al año. Por otra parte, el Marqués de la Ensenada escribió al Capitán General de Andalucía a fin de que enviase un ingeniero a Arcos encargado de elaborar el proyecto para que, una vez realizado, dictaminase junto con el Ingeniero Director Ignacio Sala, sobre la obra ${ }^{20}$.

Como ya se vió, el lugar más adecuado para la construcción era el Llano de la Caridad, dada su amplitud y su proximidad al río Guadalete en "donde los caballos pueden beber", siendo de destacar la importancia que en la época, con gran influencia de las ideas ilustradas, se da a la higiene y salud del lugar que debe ser amplio y limpio y tener el mejor acceso a aguas puras, entre otras cosas. El Cabildo solicitó que fuera un cuartel "... capaz para un reximiento de Cavalleria y con la extenzion a Poderse aquartelar un Batallon de Ynfanteria con sus Pavellones para Oficiales Quartos para sargentos y demas personas que lodevan ocupar con todos sus correspondientes ofizinas y conduzion de agua para el consumo de la tropa ..." 21 .

Surge así el proyecto de cuartel, de contorno prácticamente rectangular y que consta de dos partes: una mayor que se destina a alojamiento de soldados y a caballerizas, y otra, mas pequeña, destinada a pabellón de oficiales y oficinas. La zona izquierda, que es la mayor, presenta un patio rectangular porticado. Sus lados mayores están constituidos por la fachada y por un conjunto de ocho naves, transversales al eje mayor del patio. La parte derecha cuenta con un patio central, porticado en tres frentes y rodeado por dependencias muy compartimentadas. Su crujía de fondo comunica con un amplio corral. El sector izquierdo tiene una puerta de ingreso, en comunicación con el patio, flanqueada a derecha e izquierda por el cuerpo de guardia y el cuarto del oficial, respectivamente; el resto de la crujía lo forman un calabozo y las cocinas ${ }^{22}$. El ingreso al piso alto se hace por medio de cuatro escaleras, situadas de dos en dos, en los lados menores del patio.

19. Las cantidades solicitadas fueron de 4 maravedís en cada libra de carne, 2 en cada cuartillo de vino, 2 en cada panilla de aceite y 2 en cada cuartillo de vinagre. A.G.S. Leg. 3513.

20. Carta del Marqués de la Ensenada a la ciudad de Arcos, de 1 de septiembre de 1743. A.G.S. Leg. 3513. Es de destacar la importancia de Ignacio Sala y de su labor de magisterio, durante la primera mitad del siglo XVIII en Andalucía. El fue el inspirador y supervisor de muchos de los proyectos de construcciones militares que se hicieron en aquella época.

21. Acuerdo municipal de 29 de septiembre de 1743. A.G.S. Leg. 3513.

22. El disponer las cocinas de esta forma es típico de Bartolomé de Amphoux, ya que lo repitió en su proyecto de cuartel para Vejer de la Frontera, fechado cinco años antes que éste de Arcos. Véase MARZAL MARTÍNEZ: $O p$. Cit., p. 10. 
Este, como ya se ha dicho, tiene planta rectangular y está porticado, mediante arcos de medio punto sobre pilares de sección cuadrada. Las dependencias más amplias del frente opuesto son las ocho naves destinadas a caballerizas. Están separadas por medio de pilares y, a su vez, subdivididas en dos por seis pilares que apean arcos de medio punto. Las cubiertas pueden ser bóvedas de arista atendiendo a la disposición de los soportes. La iluminación de cada nave se hace mediante dos ventanas situadas al fondo de cada una, presentando la correspondiente al extremo izquierdo siete huecos laterales y la del flanco derecho otros cinco. El ingreso a cada una de las naves de caballerizas es independiente y se efectúa por el patio, no estando su puerta centrada, sino desplazada a la derecha, para situar junto a ella otro vano de iluminación y ventilación. Es interesante el hecho de que las dos naves extremas se dedican especialmente a caballos enfermos y a los de los oficiales, respectivamente. El piso alto tiene una disposición similar, diferenciándose de las caballerizas en varios aspectos. El primero de ellos es el de su función, ya que esta parte se destina al alojamiento de los soldados. Otra diferencia consiste en la separación entre las naves, que se realiza por muros. Las cubiertas de las naves deberían ser de madera, a dos aguas.

El sector derecho del cuartel se dispone en torno a un patio central de planta rectangular, que casi llega a ser cuadrada, y estando rodeado por una serie de dependencias, como alojamiento de oficiales, subalternos, sala de juntas, etc. El patio es también porticado, con pilares de sección cuadrada, que en el piso bajo apean arcos de medio punto y en el superior dinteles. La galería superior presenta un antepecho corrido. Las dependencias del piso bajo se cubren con techos planos mientras en el alto cuenta con un tejado a dos aguas. Tras las salas ubicadas al fondo del patio existe un amplio corral que según la leyenda del plano se utilizaba "para poner los caballos de los oficiales al fresco".

En todo el proyecto es de destacar la falta total de ornamentación, lográndose así un edificio de gran sobriedad. Lógicamente esto se debe no tanto a una evolución estilística del barroco como al hecho de ser la obra de un ingeniero militar, más interesado en los aspectos útiles y prácticos de los edificios que en su belleza formal. En este sentido, no conviene olvidar que los proyectos y construcciones del cuerpo de Ingenieros Militares facilitan la introducción en el país de las nuevas ideas constructivas y estéticas propias del clasicismo, en contra de la tradición barroca imperante hasta el momento.

Lamentablemente el proyecto de cuartel no se llevó a la práctica. En 1744 el Marqués de Pozoblanco dictaminó sobre la obra, informando al Marqués de la Ensenada que él valoraba los gastos en 643.525 reales, por lo que con el dinero presupuestado, por muy bien que se administrase, haria la obra inacabable -unos 42 años-, todo ello sin contar con los problemas que fueran surgiendo a lo largo del trabajo y que serían inevitables. Basa su afirmación en el dictamen de Igna- 
cio Sala y considera, el Marqués de Pozoblanco, que tampoco es factible construir el cuartel de infantería, por razones similares, ya que los gastos serían aproximadamente de 107.952 reales y que se tardarían unos 27 años. Concluye opinando que sí es posible realizar un cuartel para un escuadrón, disponiéndolo de forma que se pudiera ampliar en un futuro. Para levantarlo solicita permiso al Rey con objeto de aumentar los tributos y buscar un préstamo a bajo interés. Todo ello si la ciudad da su conformidad, ya que en caso contrario piensa que es imposible construir nada ${ }^{23}$. El Marqués de Pozoblanco obtuvo la autorización, pero expresamente se le indica que deben desaparecer, por encarecer la obra y no ser realmente necesarios, el alojamiento de oficiales y el puente ${ }^{24}$.

Son estas las últimas noticias documentales que se tienen sobre el proyecto. No hay noticias de que se iniciase su construcción por lo que puede afirmarse que, como en tantas otras partes de España, fueron los problemas económicos los que impidieron la realización de esta obra.

Un último aspecto a tratar es el del proyecto de puente ${ }^{25}$. Ya desde el siglo XVI, la ciudad estuvo muy interesada en la construccion de uno, porque Arcos era un lugar de "paso muy necesario para el comercio y comunicación de toda la Andalucía alta y baja y de las costas y presidios de Africa" ${ }^{26}$. Así, se observa que en el capítulo 24 de las transacciones de 1544, entre el Duque de Arcos y el cabildo municipal, se acuerda construir "un puente de obra". También se sabe que el arquitecto Hernán Ruiz II, en 1551, tuvo contactos con el concejo arcense para la construcción de un puente de piedra ${ }^{27}$. Pero no hay noticias de que se llevase a cabo, porque en 1567 aún se está hablando de cómo se repartirían las costas de la obra ${ }^{28}$. Otra prueba del afán por contar con tan útil medio de comunicación es la sesión del cabildo municipal de 3 de febrero de 1603 donde se solicitó un puente y se pidió permiso al Duque de Arcos para levantarlo ${ }^{29}$. Este lo concedió, por lo que en el cabildo de 6 de marzo de 1606 se acordó llamar a Hernán Ruiz III para la construcción del puente, ya que era considerado

23. Carta del Marqués de Pozoblanco al Marqués de la Ensenada, fechada en Málaga el 24 de marzo de 1744. A.G.S. Leg. 3513.

24. Carta del Marqués de Pozoblanco de 7 de abril de 1744. A.G.S. Leg. 3513.

25. Un estudio sobre el puente existe en BAENA GALLÉ, José Manuel: "Los proyectos de puente para Arcos de la Frontera: 1544-1920". Actas del VI Congreso Español de Historia del Arte. C.E.H.A. Los Caminos y el Arte. Tomo II: El Arte en los Caminos. Santiago de Compostela, 1989. Pp. 45-55.

26. Declaraciones de José Gómez de Mendoza en 1637. Cfr.: CUEVAS: Op. Cit., p. 22.

27. Cfr.: BANDA Y VARGAS, Antonio de la: El arquitecto andaluz Hernán Ruiz II. Sevilla, 1974. P. 174.

28. CUEVAS: $O p$. Cit., p. 22.

29. Archivo Municipal de Arcos de la Frontera (A.M.A.F.). Leg. 620 (1603-1618). Fol.: 34-39. 
como "el mejor maestro que se falla para estas cosas" ${ }^{30}$. El 12 de junio de 1606 , Hernán Ruiz informó al concejo de que "Parasacar elpilar de fundamento firme ... sean de gastar enel Edificioobra y fabrica ... por lo menos tresmill ducados" ${ }^{31}$. Se comenzaron los trabajos inmediatamente, aunque la labor quedó interrumpida, pues el 10 de julio de 1606, durante una visita a las obras, murió repentinamente el maestro ${ }^{32}$.

Las obras no continuaron, puesto que en 1650 vuelve a tratarse en el cabildo municipal de la ciudad sobre la construcción de un puente de piedra. La documentación consultada nos muestra la existencia anterior de un puente de madera en el que se hacían grandes gastos de reparación y también de una avenida del río que lo destruyó ${ }^{33}$. Por ser obra muy necesaria para la población se decidió, por acuerdo de 19 de mayo de 1650 , la construcción de uno de cantería, y para ello se solicitó la asistencia de diferentes maestros de las poblaciones cercanas, cuyo cometido sería el de seleccionar el lugar idóneo para la construcción y elevar un proyecto. Tras el estudio del lugar, se decide que el puente se debía construir donde estaba el de madera, realizando un proyecto de puente de siete ojos. Las obras se debieron comenzar, ya que se ordenó la constitución de un arca de ingresos para sufragar los gastos y la construcción de garitas de madera y cercado para custodiar las herramientas y los materiales ${ }^{34}$.

Pero tampoco se llevó a termino este proyecto, volviéndose a solicitar otro en 1674, porque se había caído el nuevo puente de madera que se usaba para el tránsito. Durante el siglo XVIII pervivió un puente de madera como se comprueba en el dibujo antes comentado, correspondiente a 1744, y por las estadísticas de 1747 , que mencionan un puente de madera libre de derechos ${ }^{35}$.

Posteriormente, en 13 de octubre de 1753, se gana Real Provisión para construir un puente de cantería. Se contrató su construcción en 1771 por 475.000 reales, comenzándose la obra y levantándose sus arranques. Pero en 1796, el Supremo Consejo de Castilla hace detener la construcción por falta de solidez ${ }^{36}$. Finalmente, en 1868 , se construye un puente de sillería que vio colmados los

30. A.M.A.F. Actas Capitulares. Leg. 620. Fol.: $138 \mathrm{rt}^{\circ}$.

31. A.M.A.F. Actas Capitulares, Leg. 620. Fol.: $140 \mathrm{vt}^{2}-151$.

32. Cfr.: BANDA Y VARGAS: $O p$. Cit. P. 219.

33. Existía desde entonces una barca que se usaba para cruzar el río. Al decidir el Cabildo Municipal la construcción de un puente de piedra, dispuso la venta de dicha barca, lo que se hizo por medio de subasta pública. A.M.A.F. Obras Públicas. Leg. 584. Expediente 5 y 6. S.f.

34. A.M.A.F. Obras Públicas. Leg. 584. Expediente 5. S.F.

35. CUEVAS: Op. Cit., p. 22.

36. MADOZ, Pascual: Diccionario Geográfico-Estadístico-Histórico de España y sus posesiones de ultramar. Madrid, 1847. Tomo II. p. 482. También nos informa que en esa época se estaba siguiendo un expediente a fin de hacer un puente colgante. 
deseos de la ciudad. Se habían tardado más de 300 años en llegar a tener un puente como lo merecían "un río y un Pueblo de tanta consideracion" ${ }^{37}$.

El diseño del puente que aparece en el proyecto que aquí se comenta corresponde, al igual que el cuartel, al ingeniero Bartolomé de Amphoux. Dicho puente constaría de tres pilares de sección hexagonal, en el cauce del río, y dos más en las orillas, cabalgando entre ellos cuatro arcos de medio punto, con ménsulas en las claves. El puente es ligeramente apuntado en el centro, por lo que los arcos centrales tienen mayor luz que los laterales. El centro del puente se corona con una cruz, y en sus accesos aparecen pedestales rematados por esferas. Hay un antepecho de obra entre ambos ingresos. El dibujo de puente que figura en el proyecto de Amphoux destaca por su simplicidad y falta de ornamentación.

En la Real Provisión de 1753 se menciona que en la década de 1740 un ingeniero militar informó sobre los restos de unos pilares que existían en la zona, probablemente pertenecientes a la obra de 1650; dicho ingeniero consideró que estos pilares estaban en perfectas condiciones y que se podía hacer sobre ellos un puente sin muchas dificultades técnicas. El ingeniero, que por la proximidad de fechas es muy posible que fuese Amphoux, formó un modelo de puente y valoró todo el gasto en 22.000 ducados, pero esta "cantidad nosiendo fasil proporsionarsela y apromptarla Dio lugar ael Abandono yOlvido deesteproyecto reservandolo paratiempo masfeliz" ${ }^{38}$. Se conoce, gracias a las Actas Capitulares del Archivo Municipal de:Arcos, que a Bartolomé de Amphoux se le pagaron 970 reales y 32 maravedís por "costas entrayda y llevadas ... yGratificazion queseledio prohavervenido a esta Ziudad a hazer la Plaza y tomar las medidas para tratar de hazer quarteles y Puente de Piedra" 39 .

Tanto la obra del cuartel, como la del puente, incluidas en el mismo presupuesto, no se pudieron llevar a cabo por problemas de tipo económico. Fueron, por consiguiente, de esta forma otro de los proyectos irrealizados en la España de los Borbones.

37. PONZ, Antonio: Viage de España. Madrid, 1792. Tomo XVII. P. 285. De todas formas el puente fue derribado por el río en 1917 y hasta 1920 no se construyó uno metálico, que es el actualmente existente.

38. Real Provisión de 13 de octubre de 1753. A.M.A.F. Actas Capitulares. Leg. 646. S.f.

39. A.M.A.F. Actas Capitulares, Leg. 643. Acuerdo de 17 de agosto de 1747. S.f. 


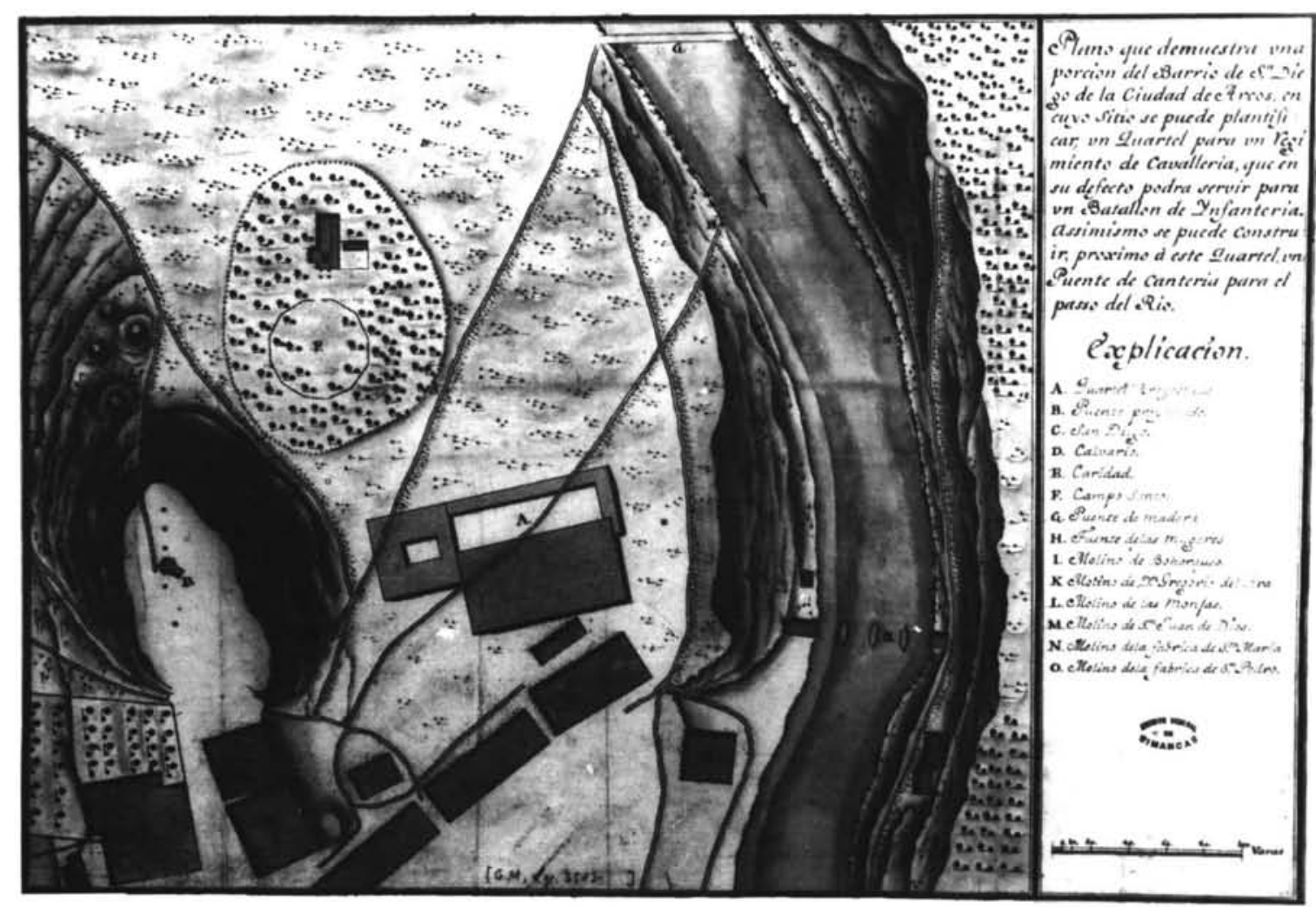

Lám. 1.

Bartolomé de Amphoux. Plano del barrio de San Diego de Arcos de la Frontera. (Archivo General de Simancas, M. P. y D. XXII-72). 


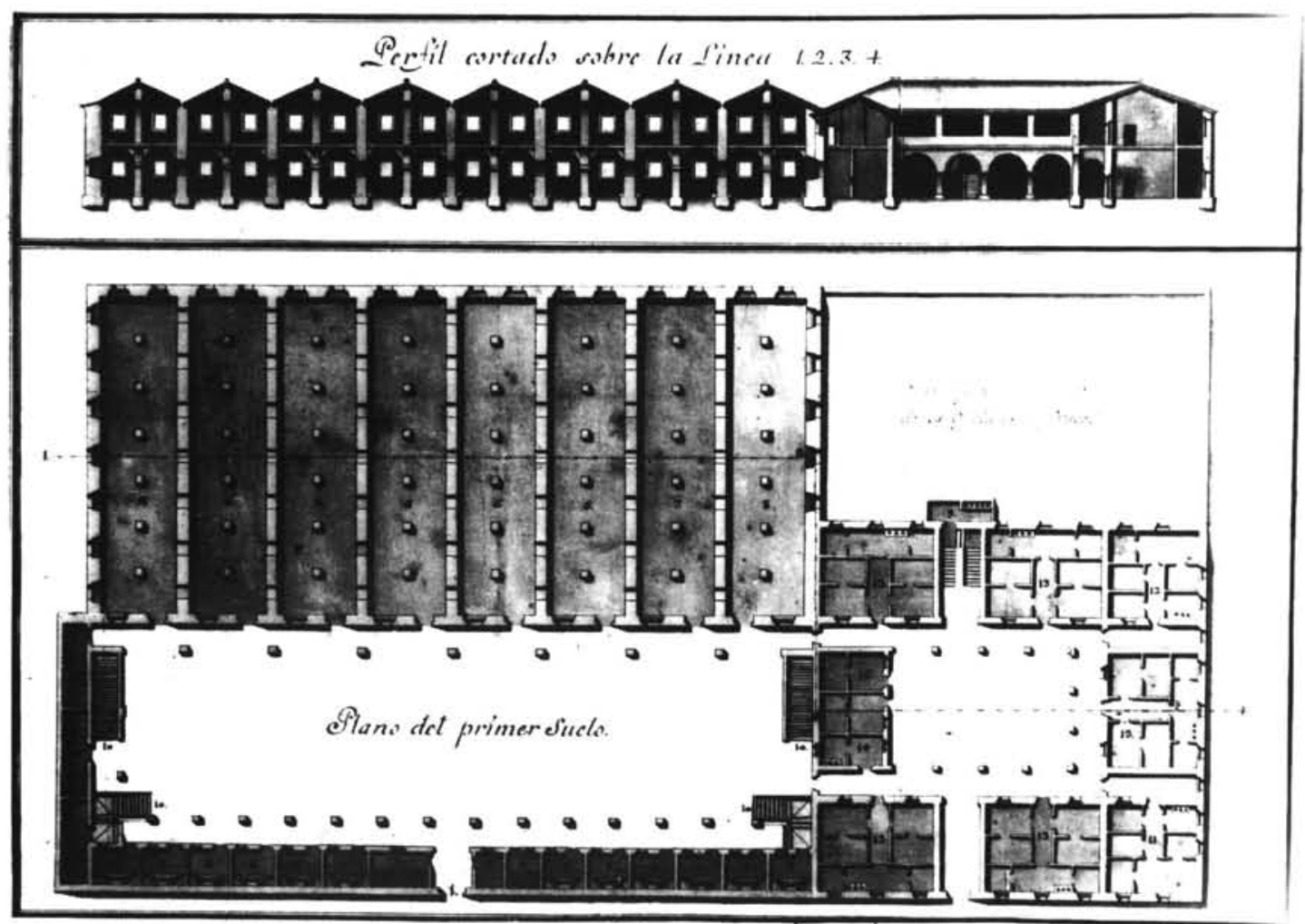

Lám. 2.

Bartolomé de Amphoux. Proyecto de Cuartel. Planta baja y Sección. (Archivo General de Simancas, M. P. y D. XXII-72). 


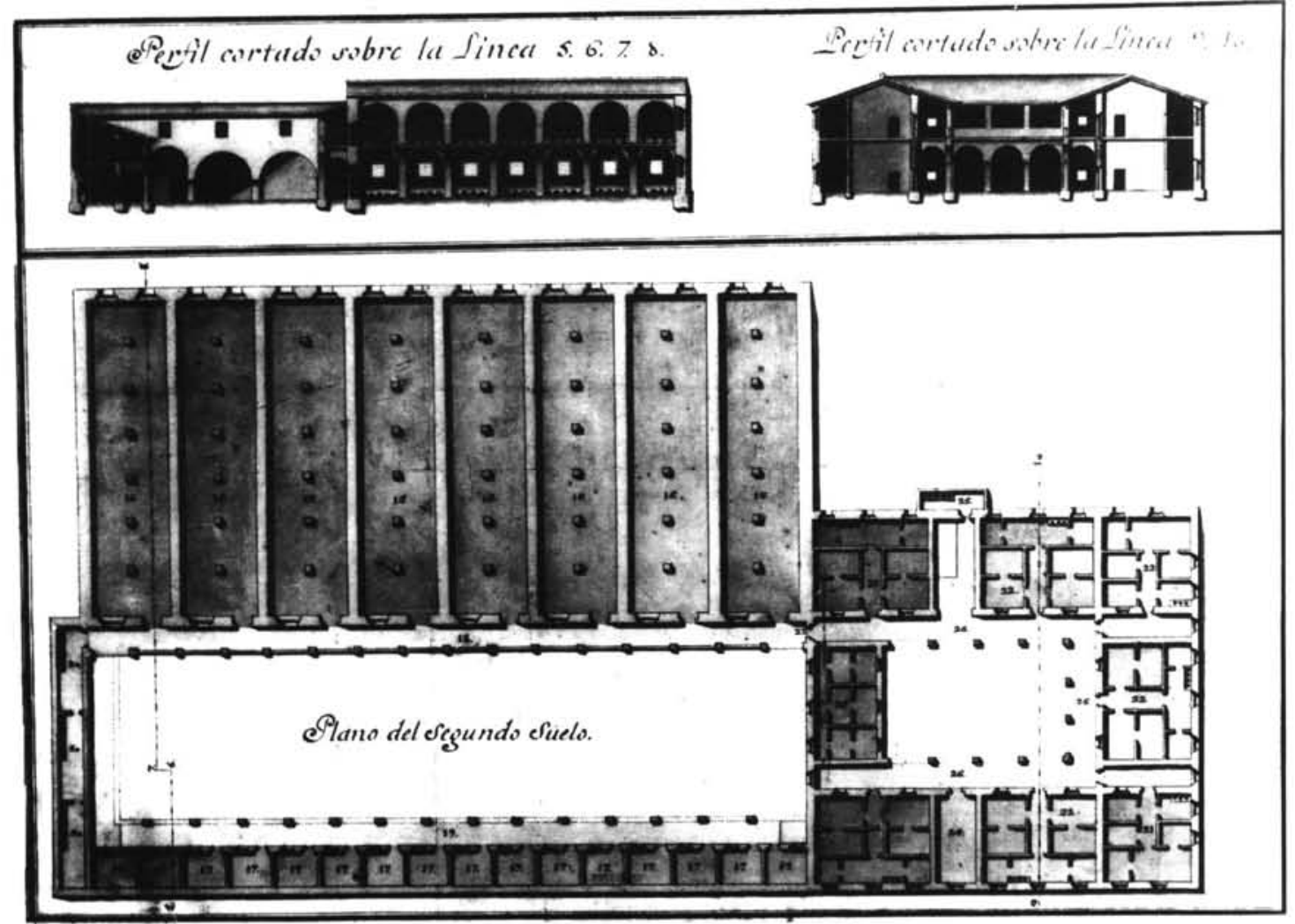

Lám. 3.

Bartolomé de Amphoux. Proyecto de Cuartel. Planta alta y Sección. (Archivo General de Simancas, M. P. y D. XXII-72). 


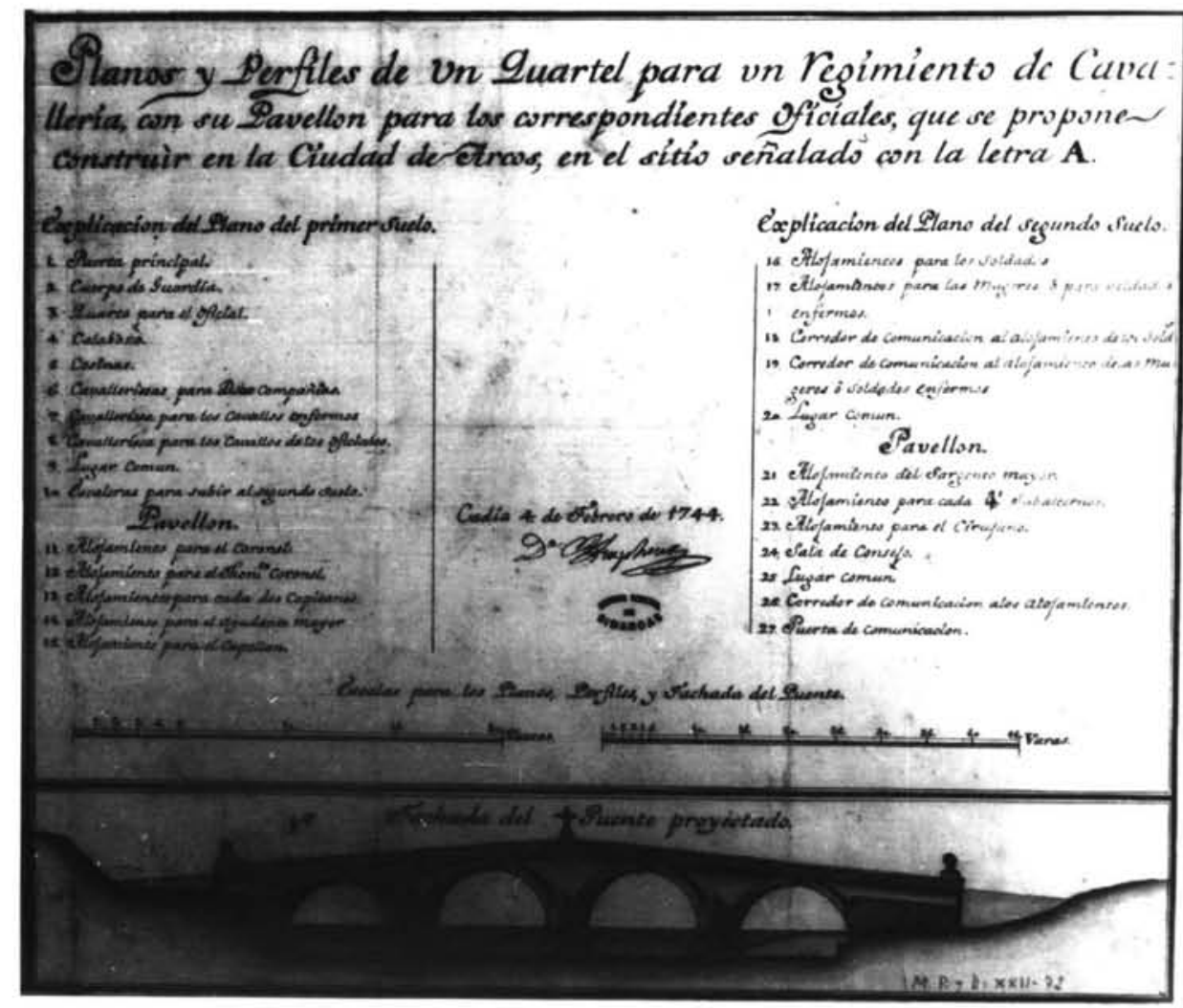

Lám. 4.

Bartolomé de Amphoux. Proyecto de Puente. (Archivo General de Simancas, M. P. y D. XXII-72) 